\title{
Optimal Placement of Multiple Interconnected Gateways in Heterogeneous Wireless Sensor Networks ${ }^{\star}$
}

\author{
Antonio Capone, Matteo Cesana, Danilo De Donno, and Ilario Filippini \\ Dipartimento di Elettronica e Informazione, Politecnico di Milano \\ Piazza L. da Vinci 32, 20133 Milan, Italy \\ surname@elet.polimi.it
}

\begin{abstract}
Data collected by sensors often have to be remotely delivered through multi-hop wireless paths to data sinks connected to application servers for information processing. The position of these sinks has a huge impact on the quality of the specific Wireless Sensor Network (WSN). Indeed, it may create artificial traffic bottlenecks which affect the energy efficiency and the WSN lifetime. This paper considers a heterogeneous network scenario where wireless sensors deliver data to intermediate gateways geared with a diverse wireless technology and interconnected together and to the sink. An optimization framework based on Integer Linear Programming (ILP) is developed to locate wireless gateways minimizing the overall installation cost and the energy consumption in the WSN, while accounting for multi-hop coverage between sensors and gateways, and connectivity among wireless gateways. The proposed ILP formulations are solved to optimality for medium-size instances to analyze the quality of the designed networks, and heuristic algorithms are also proposed to tackle large-scale heterogeneous scenarios.
\end{abstract}

Keywords: Wireless Sensor Networks, Gateway Placement, Optimization.

\section{Introduction}

Wireless Sensor Networks (WSN) have recently emerged as an ideal solution to a large number of applications where the goal is collecting measurements of a physical parameters (temperature, humidity, light intensity, etc.) or detecting events in the covered area (intrusion, wild fire, etc.) 1]. Sensors are usually low-cost battery-operated devices geared with sensing, processing and communication functionalities. Obviously, the limited energy availability deeply impacts on the design of WSN and in particular on communication protocols running at different levels [2/3|4] as well as on network deployment [5|6] and topology.

^ This paper has been partially supported by research project PRIN SESAME.

L. Fratta et al. (Eds.): NETWORKING 2009, LNCS 5550, pp. 442 455, 2009.

(C) IFIP International Federation for Information Processing 2009 
In many scenarios, data collected by sensors must be delivered to application servers for processing. These servers can be reached via a sink node that is connected to a local or geographical network through a communication interface with a different technology with respect to the WSN. Since the transmission range of sensor nodes is often much smaller than the area covered by the WSN, sensors cooperate to deliver information to the sink node through multi-hop paths.

Multi-hop information delivery to sink nodes requires multiple transmissions that consume energy in intermediate nodes and heavily limit lifetime of those nodes that are used more frequently. Even if many routing approaches have been proposed to balance the load among nodes and prolong the overall network lifetime, it is quite evident that nodes closer to the sink are anyway the most critical ones since they are required to relay all traffic generated by the other nodes and headed to the sink. For this reason, several methods have been proposed for optimizing the sink position in WSN design [13 15.

However, a single sink node collecting all data from the network is not for sure an efficient solution when dealing with large size WSNs, like e.g. in applications for monitoring natural areas. Indeed, as the network grows, the amount of information that must be delivered to the sink by surrounding nodes increases creating traffic and energy consumption bottlenecks. A promising alternative approach is based on the use of additional concentration nodes, gateways, that allow to spread the load and also to keep multi-hop paths within a reasonable length 81010. Also in this case gateway positioning is a key element for designing efficient networks. The entire set of sensor nodes need to be divided into independent subsets that send their traffic to a gateway, giving rise to a clustering problem.

Generally speaking, gateways are more powerful and expensive devices with respect to sensors and a reasonable objective is minimizing their number while matching constraints on energy efficiency and network quality 81012. However, this approach focuses on the WSN only neglecting the problem of interconnecting the gateways to the sink or more in general to the network where application servers are located. According to the specific application scenario, several solutions can be adopted for this problem, including the use of direct geographical links among the gateways (wired links, satellite links, etc.). However, this may be quite expensive and inefficient, since the traffic collected at each gateway is usually much smaller than the link capacity while its cost (interface, installation, and operation) often high.

For this reason, in this paper we consider an alternative network scenario where gateways are inter-connected through a Wireless Mesh Network (WMN) and act as Wireless Routers (WRs) forwarding traffic along multi-hop paths toward a sink node that is the only one equipped with a geographical link (see Figure 1). The resulting architecture is an heterogenous multi-hop network with different devices (sensors, MRs, and access point) and wireless links (low-power low-rate links for sensors, and high rate links for mesh routers) that can be 


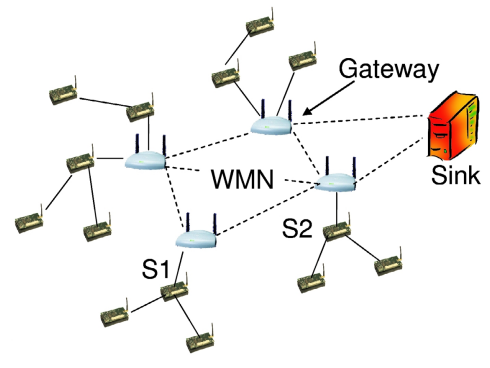

Fig. 1. Reference Network Scenario

adapted to the specific application scenario using available technologies (like e.g. ZigBee, WiFi, WiMax, etc.).

In this work we investigate the joint problem of selecting/positioning the gateways and designing the WMN that interconnects them. We provide an optimization framework that minimizes installation costs and maximizes the energy efficiency, while considering both multi-hop coverage and connectivity constraints. Coverage considers the availability of a multi-hop path between each sensor node and at least a gateway, while connectivity among gateways must be ensured by properly designing the WMN. The proposed optimization framework is independent on the specific routing strategy in the WSN; indeed, routes in the WSN (from sensors to gateways) are assumed to be given. This allows to use any of the routing mechanisms that have been proposed for WSNs [3] and to plug it into our optimization framework just modifying a separate software module that compute traffic loads in the WSN. We note here that routing could be easily optimized together with gateway positions by including further degrees of freedom (variables) in the optimization framework 812. However, we believe that our approach is more practical since in real-life wireless sensor networks the network installer can hardly modify/optimize the routing schemes running in the specific WSNs technology.

We propose an Integer Linear Programming (ILP) framework that allows to solve to optimality reasonable size networks and to evaluate the impact of system parameters, including the routing strategy, on the obtained solutions. We also propose an heuristic approach that allows to achieve good quality solutions (within 10\% from the optimum in the considered scenarios) in short computing time, tackling also the optimization of large-scale networks.

The paper is organized as follows. In Section 2 we provide a detailed description of the the Multiple Interconnected Gateway Placement (MIG-P) problem and we present our ILP framework to formulate the problem. Section 3 provides numerical results to get insight on the characteristics of the optimal solutions obtained through the ILP framework. The heuristic algorithms proposed for tackling large size instances and results to assess their performance are presented in Section 4. We review and discuss previous works on this topic in Section 5 and conclude the paper in Section 6 . 


\section{The Multiple Interconnected Gateway Placement Problem}

We consider a heterogeneous network scenario where clouds of Wireless Sensor Networks (WSNs) for data collection are connected to a sink node through a wireless mesh backbone of gateway devices. We will use gateways and WRs as synonyms throughout the paper. Each WR acts as a traffic concentration point for all the sensor nodes belonging to the corresponding WSN. In such network scenario, the number and the positions of the WRs obviously impact on the wireless sensor network lifetime. In fact, roughly speaking, placing a WR in a given position increases the load of all the sensors in the one-hop neighborhood, which are forced to relay to the gateway also the traffic coming from the leaves sensors. We will call these sensor nodes critical nodes with respect to a given WR (e.g., nodes S1 and S2 in Fig. 1 are critical nodes).

In this work, we aim at optimizing the overall network topology by properly selecting number and locations of WRs in such a way that installation cost are minimized and energy efficiency (network lifetime) maximized, under the constraints that each sensor must be connected through a multi-hop path to the closest WR (to limit energy consumption due to packet forwarding), and that a multi-hop path does exist also between each WR and the traffic sink. Therefore, the optimization framework jointly considers the selection/positioning of WRs, as well as the design of the WMN among WRs.

We take a constructive approach in presenting the mathematical programming framework. Indeed, we introduce first a basic version of the MIG-P problem which considers only installation cost minimization while ensuring coverage within a maximum number of hops of all the sensors and connectivity between gateways and sink node; finally, we extend the basic version to an advanced version which also minimizes the load of critical nodes.

The WSN can be represented as a set of test points $\mathcal{S}=\left\{1 . . N_{S}\right\}$ in the network, whereas a set of candidate sites, denoted by $\mathcal{W}=\left\{1 . . N_{W}\right\}$, defines the potential positions where WRs can be installed. Each WR can establish a wireless link with any other WR at a distance smaller than its communication range $R_{W}$ on the backbone, as well as with any sensor at distance lower than $R_{C}$ in the WSN 1 . According to that, we define an adjacency matrix that summarizes connectivity among candidate sites:

$$
I_{i j}=\left\{\begin{array}{l}
1 \text { if } i \in \mathcal{W}, j \in \mathcal{W}, \operatorname{dist}(i, j) \leq R_{W} \\
0 \text { otherwise }
\end{array}\right.
$$

where $\operatorname{dist}(\cdot)$ is the Euclidean distance between the positions of the two involved devices.

The routing pattern is an input parameter of the optimization problem. Given a candidate site, the specific routing strategy adopted in the WSN determines

\footnotetext{
${ }^{1}$ The link definition on the basis of a communication range is only for the sake of presentation. We note here that the proposed framework holds for any type of connectivity matrix.
} 
the assignment of sensor nodes to gateways, as well as the set of multi-hop paths used for collecting data. To account for the pre-computed routes, we introduce matrix $R$ which represents the pre-computed routing trees rooted at each WR:

$$
R_{i j}^{c}=\left\{\begin{array}{l}
1 \text { if link }(i, j), i, j \in \mathcal{S}, \text { belongs to the tree rooted at WR } c \\
0 \text { otherwise }
\end{array}\right.
$$

To further represent the concept of multi-hop coverage, we introduce the following binary matrix $C$, which indicates whether each sensor is connected through a multi-hop path to a given WR:

$$
C_{i j}=\left\{\begin{array}{l}
1 \text { if there exists a route from sensor node } i \text { and WR } j \\
\text { with no more than } M A X_{H O P S} \text { hops } \\
0 \text { otherwise }
\end{array}\right.
$$

being $M A X_{H O P S}$ is the maximum allowed number of hops of a route.

Finally, for each sensor node $i$ we define the ordered vector $O_{i}$ of the reachable WRs. The ordering relation is such that given two WRs, one at the $j$-th place, $O_{i}(j)$, and one at the $k$-th, $O_{i}(k)$, if $j<k$ then $\operatorname{dist}(i, j) \leq \operatorname{dist}(i, k)$. Sets $\mathcal{J}_{i}$ are index sets of vectors $O_{i}$.

We introduce the following sets of decision variables defining WR installation, node-gateway assignment and routing in the WMN. In detail:

$$
\begin{gathered}
y_{i}=\left\{\begin{array}{l}
1 \text { if a WR is installed in candidate site } i \\
0 \text { otherwise }
\end{array}\right. \\
x_{i j}=\left\{\begin{array}{l}
1 \text { if sensor node } i \text { is assigned to WR } j, \\
\text { that is, node } i \text { routes its traffic toward WR } j \\
0 \text { otherwise }
\end{array}\right.
\end{gathered}
$$

$f_{i j}$ are integer flow variables for links $(i, j), i, j \in \mathcal{S}$ in the WMN formed by installed WRs, while the $f_{w}$ represents the overall traffic entering the sink node.

The proposed ILP model formulation for the basic version of the MIG-P problem is:

$$
\begin{aligned}
& \min \sum_{i \in \mathcal{W}} y_{i} \\
& \text { s.t. } \\
& \quad \sum_{j \in \mathcal{W}} x_{i j}=1 \quad \forall i \in \mathcal{S} \\
& x_{i j} \leq C_{i j} y_{j} \quad \forall i \in \mathcal{S}, j \in \mathcal{W} \\
& \quad \sum_{i \in \mathcal{S}} x_{i j}+\sum_{l \in \mathcal{W}}\left(f_{l j}-f_{j l}\right)=\left\{\begin{array}{c}
f_{w}, \text { if } j=S I N K \\
0, \quad \text { otherwise }
\end{array} \quad \forall j \in \mathcal{W}\right. \\
& f_{i j} \leq N_{S} I_{i j} y_{i}, f_{i j} \leq N_{S} I_{i j} y_{j} \quad \forall i, j \in \mathcal{W} \\
& y_{O_{i}(k)}+\sum_{h \in \mathcal{J}_{i}, h>k} x_{i O_{i}(h)} \leq 1 \quad \forall i \in \mathcal{S}, k \in \mathcal{J}_{i} \quad \\
& \\
& y_{i} \in\{0,1\}, x_{h j} \in\{0,1\}, f_{i j} \in \mathbb{Z}^{+}, f_{w} \in \mathbb{Z}^{+} \quad \forall i, j \in \mathcal{W}, \forall h \in \mathcal{S}
\end{aligned}
$$


where $S I N K$ is the WR chosen as sink node (MAP). Objective function (10) states the minimization of the WR installation cost. In this formulation all candidate sites have the same installation cost, however, it can be easily extended to consider a different cost for each site. Constraints (2) and (3) impose that each sensor node is covered by an installed WR. Constraints (4) are flow balance constraints defining the routing among WRs, while constraints (5) allow the traffic flowing through a link only if the two extreme WRs are installed. Constraints (6) force each sensor node to be assigned to the closest installed WR. Finally, constraints (7) define the domain of each variable.

The second version of the MIG-P problem directly accounts for load balancing and energy efficiency, by minimizing the number of concurrent flows travelling through of the most loaded nodes around the WRs (critical nodes). The set of critical nodes can be denoted by $\mathcal{S}^{C} \subset \mathcal{S}$, whereas $\mathcal{W}_{i}^{C} \subset \mathcal{W}$ is the set of candidate sites for which a sensor node $i$ is a critical node. The number of paths through a critical node $i$ is given by variable $p_{i}, \forall i \in \mathcal{S}^{C}$. The new ILP model consists in replacing objective function (1) with the following:

$$
\min \sum_{i \in \mathcal{W}} y_{i}+\alpha \sum_{j \in \mathcal{S}^{C}} p_{j}
$$

and including the additional constraints:

$$
\begin{aligned}
& \sum_{j \in \mathcal{S}: j \neq i} R_{j i}^{c} x_{j c} \leq M_{P} x_{i c}+p_{i} \quad \forall i \in \mathcal{S}^{C}, c \in \mathcal{W}_{i}^{C} \\
& p_{i} \in \mathbb{Z}^{+} \quad \forall i \in \mathcal{S}^{C}
\end{aligned}
$$

where $M_{P}$ is a threshold on the number of paths going through a critical node: when this number is greater than $M_{P}$ the critical nodes is considered overloaded. These constraints enforce the number of actually used routing paths through a critical node $i$ (left term) to be less or equal to the overload threshold $M_{P}$. Note that (9) does not define a hard constraint, but, on the other hand, if the overload threshold is violated, the variable $p_{i}$ records the violation.

The new objective function (8) aims at minimizing both the number of installed WRs and the overload of critical nodes. The scalar $\alpha$ is a conversion parameter between the cost of installing a WR and the overload of critical nodes. The amount of overload recorded in $p_{i}$ penalizes the objective function and can be actually mapped into the cost of additional power supply for node $i$ (f.i., recharging costs, high capacity batteries, etc.). We have experimentally set $\alpha$ equal to 0.1 .

\section{Optimal Gateway Placement}

We have tested the quality of the planned heterogeneous WSNs under two different routing algorithms: a classical min-hop shortest path, and an ideal energyaware routing. In the latter case, routing paths are obtained solving a Minimum Cost Flow problem towards each candidate site, where the the best routing paths from every sensor to a given candidate site are determined according to the following objective function: $\min \sum_{(i, j) \in \mathcal{L}} \operatorname{dist}(i, j)$ flow $_{i j}+\beta m^{C}$, where $\mathcal{L}$ is the set 
of wireless links among sensor nodes and the gateway located at the site, flow $w_{i j}$ are variables defining the number of node-gateway paths through link $(i, j)$ and $m_{C}$ expresses the number of routing paths going through the most loaded critical node. Note that the obtained routes preserve a shortest path nature but, at the same time, guarantee an even load distribution among critical nodes. Once the routing is defined, matrices $R$ and $C$ can be fed into the formulations to be solved in reference scenarios.

We consider here randomly deployed sensors and candidate sites over a square area with edge in the range $250 \mathrm{~m}-350 \mathrm{~m}$. The communication ranges for sensors and $\mathrm{WRs}$ are $20 \mathrm{~m}$, and $100 \mathrm{~m}$, respectively. The maximum route length parameter $M A X_{H O P S}$ varies from 3 to 5. The problem is modelled with AMPL 21] and solved with the CPLEX 10.0 solver 22 on INTEL $3.2 \mathrm{GHz}$ machines with $2 \mathrm{~GB}$ of RAM operated by Linux. Each result has been averaged on 10 instances.

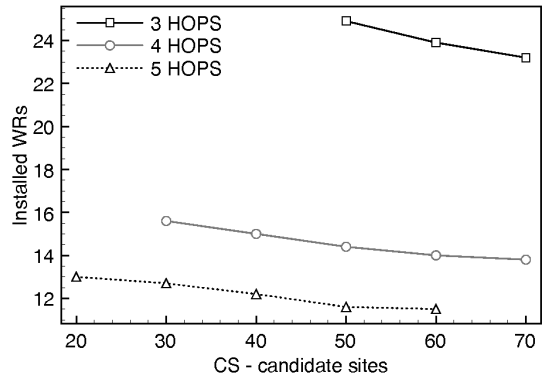

(a) Candidate sites

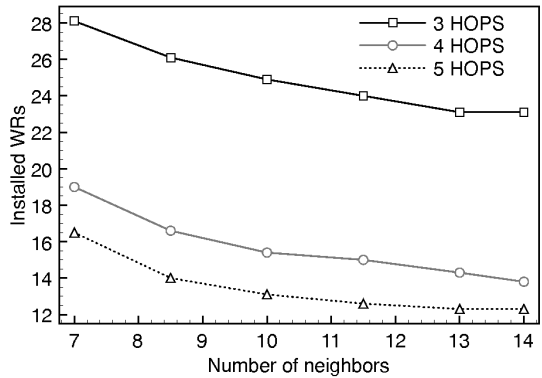

(b) Deployment density

Fig. 2. Installed MRs vs. the number of candidate sites (a) and deployment (b). Area $300 \mathrm{~m} \times 300 \mathrm{~m}$ and $M A X_{H O P S}=3,4,5$. a) 1000 sensors; b) from 500 to 1000 sensors, 60 candidate sites.

Figure 2(a) shows the average number of installed WRs when varying the number of candidate sites and the maximum path length. As the number of candidate sites increases, the solution space enlarges and, therefore, the solver can select better options reducing the overall installation cost. As for the maximum path length, achieving coverage is more difficult with shorter paths and cost increases since additional WRs must be installed to provide multi-hop coverage to isolated sensors. We observe that the network installation cost halves when passing from $M A X_{H O P S}=3$ to $M A X_{H O P S}=5$. Figure 2(b) shows the average number of installed WRs vs. the density of sensor nodes (expressed as number of neighbors). As expected, the installation cost decreases as the sensor density increases since more multi-hop paths between sensors and WRs area available and coverage is easier.

In order to assess the importance of modelling the energy efficiency directly into the optimization problem, we compare the solutions obtained with the basic version of the gateway placement problem against the advanced one. Some results on the load distribution of critical nodes are reported in Figures 3(a) and 3(b) 
which refer to the minimum hop routing, and the ideal energy-aware routing algorithm, respectively. Plots show the percentage of critical nodes crossed by the number of routing paths reported on x-axis. For a given critical node, the number of crossing paths corresponds to the number of incoming traffic flows to be forwarded to the closest installed WR. We observe that the advanced formulation provides a larger fraction of critical nodes with less than $M_{P}$ paths $\left(M_{P}=3\right.$ in the figure), that is, a larger fraction of not overloaded critical nodes. The gap between the two curves is larger when the energy-aware routing is used as it allows to spread the load among critical nodes.

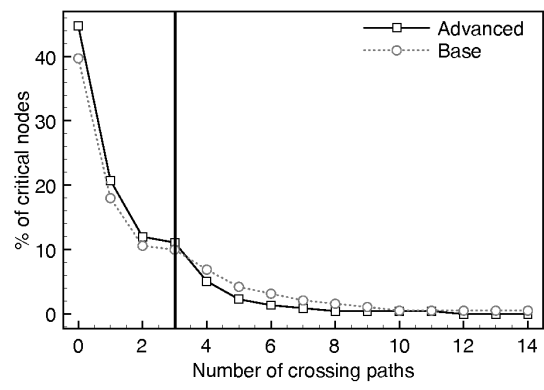

(a) Min-hop routing

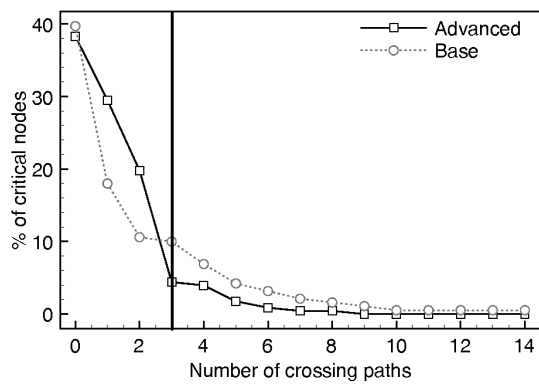

(b) Energy-aware routing

Fig. 3. Comparison of the basic and advanced formulations according to the load distribution among critical nodes for both min-hop and energy-aware routing. Area $300 \mathrm{~m}$ x $300 \mathrm{~m}, 1000$ sensor nodes, 70 candidate sites, and $M A X_{H O P S}=4$.

Table 1. Comparison among different problem formulations. Area $300 \mathrm{~m}$ x 300m, 1000 sensor nodes, 70 candidate sites, and $M A X_{H O P S}=3$.

\begin{tabular}{|c|c|c|c|}
\hline Formulation & Routing & $\begin{array}{c}\text { Average Load } \\
\text { of critical nodes }\end{array}$ & Installed WRs \\
\hline \hline Basic & Min-hop & 2.75 & 23.2 \\
\hline Advanced $M_{P}=3$ & Min-hop & 2.37 & 25.2 \\
\hline Advanced $M_{P}=0$ & Min-hop & 2.23 & 29.2 \\
\hline Advanced $M_{P}=3$ & Energy-aware & 1.97 & 26.9 \\
\hline
\end{tabular}

In Table 1 we summarize the average results for the number of installed WRs and the load of critical nodes with possible combinations of problem versions and routing algorithms. Note that the results with $M_{P}=0$ refer to the minimization of the total load of critical nodes, and not only the load of the overloaded ones. Results confirm that the best solutions are obtained with the advanced problem formulation and the energy-aware routing. Moreover, with min-hop routing the lower routing efficiency needs to be compensated by a high number of gateways.

Figures 4(a) and 4(b) report two snapshots of planned networks with different $M A X_{H O P S}$. We reported the Voronoi diagrams representing the WSNs clouds 


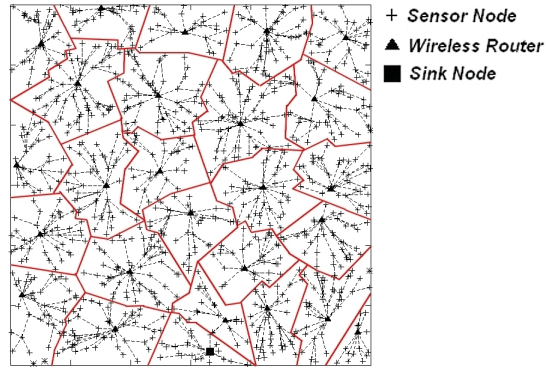

(a) $M A X_{H O P S}=3$

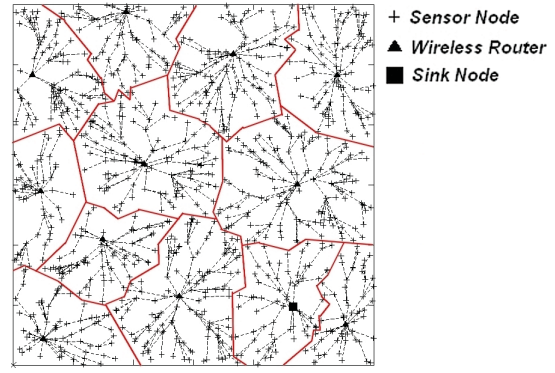

(b) $M A X_{H O P S}=5$

Fig. 4. Network Planned with the advanced version model. Area $300 m$ x $300 m, 1000$ sensor nodes, 70 candidate sites.

around each installed gateway that allow to easily observe that increasing the $M A X_{H O P S}$ leads to larger clusters.

\section{Heuristic Algorithms}

It is easy to show that the MIG-P problem is NP-hard since it contains the Minimum Cardinality Set Covering problem as sub-problem. Even if we have shown that the problem can be solved for reasonable size instances, we have also designed heuristics to cope with large networks. We present first an algorithm for the basic version of the MIG-P problem, and then we extend it to solve the advanced version.

Our heuristic approach is based on a continuous relaxation of the corresponding ILP model, and it can be summarized in the following three steps.

STEP 1 - Continuous relaxation. The ILP model (1)-(7) is solved relaxing the integrality of variables $y_{j}$ (WR installation) and $x_{i j}$ (node-WR assignment). Experimentally, we noted that about $60-70 \%$ of relaxed variables are integer (with $10^{-9}$ precision), thus, we set the value of these variables to 1 or 0 . Other variables are rounded in the following step.

STEP 2 - Variable rounding. It consists of a greedy rounding over variables $y_{j}:$

- We generate an ordered list of candidate sites associated to fractional variables $y_{j}$. The top-list candidate site is the one covering the largest number of sensor nodes.

- We scroll down the list starting from the top. For each site, we round to 1 the associated variable until full coverage is achieved. At this point the coverage requirement is satisfied, but the connectivity one may be not.

- We generate a new ordered list of the candidate sites not yet considered: the first item is the candidate site with the smallest number of installed WRs in its neighborhood. In other words, the head of the list is the most isolated candidate sites. 
- Again, we scroll the list rounding to 1 the associated variables, until the WMN is connected. At the end of this step coverage and connectivity requirements are satisfied, thereby remaining fractional variables are set to 0 . The result is an initial feasible solution to be refined in STEP 3 removing WRS in excess.

STEP 3 - Refinement This step removes as many installed WRs as possible without violating coverage and connectivity constraints. The installed WRs are ordered according to the non-increasing number of neighboring WRs. We scroll the list iteratively removing WRs. At each WR, if the solution is still feasible after its removal, we keep the new solution, otherwise, we re-insert the WR. When the end of the list is reached, the last feasible solution is the final one. Finally, the node-WR assignment is obtained running the ILP model where variable $y_{i}$ are now parameters set according to the above-described algorithm.

Figures $5(\mathrm{a})$ and $5(\mathrm{~b})$ show the average optimality gap of the heuristic algorithm under different numbers of sensor nodes and candidate sites. As expected, the quality of the heuristic solution slightly decreases when the solution space increases (candidate sites number increases). However, the largest gap is way below $10 \%$.

As for the advanced version of the problem, the workflow of the heuristic does not change: STEP 1 solves the relaxed ILP model for the advanced formulation, while STEP 2 and STEP 3 provide a feasible initial solution. Only one further step, STEP 4, has been added to the previous framework.

STEP 4 - WR re-positioning The step that most significantly impacts on the result of the algorithm is STEP 1, which fixes values of $60-70 \%$ of the overall variables. However, since assignment variables $x_{i j}$ are relaxed during STEP 1, constraints (9) on the load of critical nodes cannot be properly evaluated. Consequently, after STEP 3, we have solutions with a small number of installed WRs, but with a poor quality in terms of energy consumption. The aim of this STEP 4 is to re-position installed WRs in order to decrease the load of critical nodes. We iteratively perform the following move:

- We place each installed WR in one of the free candidate sites $\left(y_{j}=0\right)$ in its neighborhood. At each iteration, coverage and connectivity requirements are verified. If the solution is feasible and it decreases the load of critical nodes (the second term of the objective function (8) ) with respect to the last feasible solution, then the new solution is kept.

This move is repeated until an improving solution cannot be found or a maximum number of iterations is reached.

We remark here that STEP 4 does not change the number of installed WRs which are just re-positioned in order to achieve a better load balancing among critical nodes. The optimality gap of this approach, not reported here for the sake of brevity, has a very similar behavior to the one reported in Figures 5 (a) and 5(b), Again, the gap is upper bounded by $10 \%$ in all the cases under analysis.

We have tested the heuristic approaches also in large scale heterogeneous WSNs with 1000-3000 deployed sensor nodes and 50-200 available candidate 


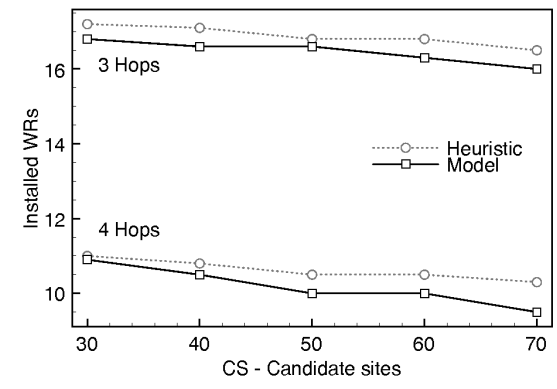

(a) Area $250 \mathrm{~m} \times 250 \mathrm{~m}, 700$ sensor nodes and $M A X_{H O P S}=3,4$

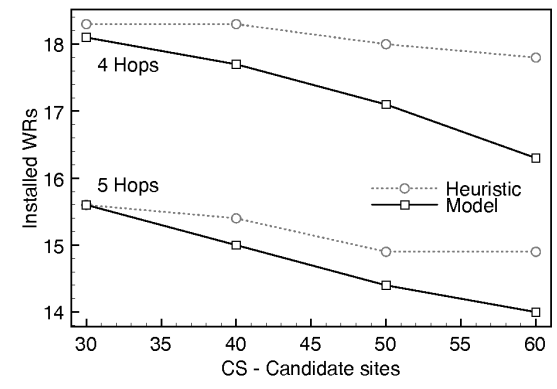

(b) Area $300 \mathrm{~m} \times 300 \mathrm{~m}, 1000$ sensor nodes and $M A X_{H O P S}=3,4$

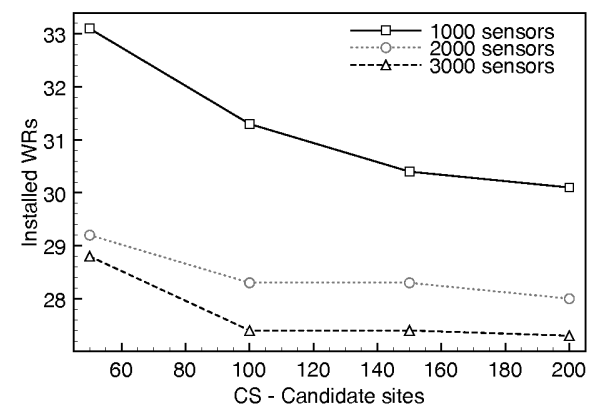

(c) Area $350 \mathrm{~m} \times 350 \mathrm{~m}$ and $M A X_{H O P S}=3$

Fig. 5. Installed WRs as the number of candidate site changes using heuristic algorithms

sites in a square area $350 \mathrm{~m}$ x $350 \mathrm{~m}$, which corresponds to a density of 10-30 neighbors within the communication range. The corresponding numerical results are shown in Figure 5(c) and confirm the same performance trend highlighted by the optimal solutions.

\section{Related Work}

The Gateway Placement problem has been extensively studied in literature. The whole set of works can be divided into two classes. One is the class of Static Base Station Positioning problems where the WSN is deployed, gateways are installed and their positions never change for the entire network lifetime. The other class, the class of Dynamic Base Station Positioning problems is characterized by the gateway re-positioning during the network operation. The latter type of problems investigate the way of moving gateways to react in front of topology changes, failures, new QoS parameters or simply to balance the energy consumption among sensor nodes. Even though many works of this class exist 111820, this type of problems is out of the scope of our work. 
We focus on the class of Static Base Station Positioning problems. Some approaches consider the best placement of a single sink node. In [13] authors propose an iterative repositioning geometric algorithm to find the best sink location in terms of minimum overall transmission energy. However, they assume the gateway is always reachable within a single hop. General sensor-sink paths are considered in [15] where an approximated algorithm aims to maximize the lifetime of a single-sink WSN.

When more gateways are available the problem gets harder because both positions and number of installed gateways must be chosen. The installation of multiple gateways induces the partition of the WSN into clusters, each one assigned to a gateway. Authors in [16] study the problem of positioning the smallest number of gateways in such a way sensors are covered in a single hop by gateway and the set of gateways forms a connected network. They propose a solution approach that divides the deployment area in square cells, finds the best single gateway placement for each cell, and then connects cells installing, if required, new gateways. It does not include any energy issue and lack in multihop paths between sensors and gateways.

In [10] heuristic algorithms based on local search and greedy techniques are proposed. Their objective is finding the best sensor nodes to be selected as gateways. The selection of the fixed number of gateways aims to minimize the overall energy consumption. Authors in 9] propose ILP models. They define several versions of the Gateway Placement problem that minimize the number of gateways with a coverage requirement or minimize path lengths from nodes to gateways with an installation budget and coverage constraints. However, these approaches do not guarantee connectivity among gateways and/or pay not attention to the load of critical nodes.

The work in [17] present ILP models where energy issues are expressed as limits on the cluster size and the number of hops between a node and a gateway. Authors in 8 propose an ILP model. The objective is minimizing the maximum number of transmitted/received packets at each sensor node. Given a fixed number of gateways, the solution provides optimum gateway placement and flows along links. The work in 12 presents Linear Programming (LP) models that maximize the total throughput during the network lifetime with fairness guarantees. Solutions provide gateway positions and data rate values. The last three modeling aproaches, besides not address gateway connectivity, force the selection of optimized routing paths.

Some further works start from a planned network and try to improve the topology according to some energy-aware principles. Although they do not consider an actual placement problem, they are interesting because of their approaches to energy-aware sensor clustering. Authors in [14] consider the problem of adding a given amount of energy to the WSN, expressed as gateways to be properly located in the area, in order to extend the network lifetime. In [7] a node-gateway assignment algorithm is proposed. It aims at distributing evenly the number of nodes among gateways. In [19], instead, the assignment algorithm is a genetic algorithm aiming at minimizing the total number of hops within the network. 


\section{Conclusion}

In this paper we have proposed an optimization framework for the design of WSNs where gateways must be placed in the area and interconnected with the sink through the wireless links of a WMN. The resulting network architecture is an heterogeneous multi-hop wireless networks where short-range low-rate links interconnect sensor nodes, while long-rage high-rate links create a wireless backbone among gateways.

The objective is that of optimizing a tradeoff between the network installation cost (the number of gateways if their installation cost is the same) and the energy consumption which is modelled considering the load of nodes directly connected to the gateways (critical nodes). The gateway selection is subject to multi-hop sensor coverage constraints that guarantee that each sensor can reach a gateway within a maximum number of hops. Moreover, the wireless network among gateways must be connected so as to allow them to deliver data to the sink.

The proposed solution approach is based on ILP models that allow to obtain optimum solutions for instances of reasonable size. Our problem formulation can consider any type of routing mechanism in the WSN and just account for its effect on the network energy efficiency. This is a key issue since in most application scenarios it is absolutely not possible to enforce a routing scheme optimized during the network design phase as it is constrained by the wireless technology and protocols adopted.

We have analyzed the effect of several parameters on the optimal solutions obtained via the ILP formulations and showed that the coverage constraints and the routing scheme can greatly impact the overall network cost and energy efficiency.

Furthermore, to tackle large problem instances we developed heuristic algorithms based on the continuous relaxation of ILP models. We showed that our heuristics provide good sub-optimal solutions (within 10\% from the optimum) in short time and allow planning very big networks with thousands of nodes.

\section{References}

1. Akyildiz, I.F., Su, W., Sankarasubramaniam, Y., Cayirci, E.: Wireless Sensor Networks: A Survey. Comp. Netw. 38, 393-422 (2002)

2. Demirkol, I., Ersoy, C., Alagoz, F.: MAC protocols for wireless sensor networks: a survey. IEEE Comm. Mag. 44(4), 115-121 (2006)

3. Akkaya, K., Younis, M.: A survey on routing protocols for wireless sensor networks. Ad Hoc Networks 3(3), 325-349 (2005)

4. Campelli, L., Capone, A., Cesana, M., Ekici, E.: A Receiver Oriented MAC Protocol for Wireless Sensor Networks. In: IEEE MASS 2007, Pisa, Italy (October 2007)

5. Cardei, M., Wu, J.: Energy-efficient coverage problems in wireless ad-hoc sensor networks. Comp. Comm. 29(4), 413-420 (2006) 
6. Amaldi, E., Capone, A., Cesana, M., Filippini, I.: Coverage planning of Wireless Sensors for Mobile Target Detection. In: IEEE MASS 2008, Atlanta, Georgia (October 2008)

7. Gupta, G., Younis, M.: Load-Balanced Clustering of Wireless Sensor Networks. In: IEEE ICC 2003, Anchorage, Alaska (May 2003)

8. Gandham, S.R., Dawande, M., Prakash, R., Venkatesan, S.: Energy Efficent Schemes for Wireless Sensor Networks with Multiple Mobile Base Stations. In: IEEE GLOBECOM 2003, San Francisco (December 2003)

9. Wong, J.L., Jafari, R., Potkonjak, M.: Gateway placement for Latency and Energy Efficient Data Aggregation. In: IEEE LCN 2004, Tampa, Florida (November 2004)

10. Bogdanov, A., Maneva, E., Riesenfeld, S.: Power-Aware Base Station Positioning for Sensor Networks. In: IEEE INFOCOM 2004, Hong Kong (March 2004)

11. Akkaya, K., Younis, M., Bangad, M.: Sink Repositioning for Enhanced Performance in Wireless Sensor Networks. Comp. Netw. 49(4), 512-534 (2005)

12. Kim, H., Seok, Y., Choi, N., Choi, Y., Kwon, T.: Optimal Multi-sink Positioning and Energy-efficient Routing in Wireless Sensor Networks. In: IEEE ICOIN 2005, Jeju Island (Febuary 2005)

13. Pan, J., Cai, L., Hou, Y.T., Shi, Y., Shen, X.: Optimal Base-Station Locations in Two-Tiered Wireless Sensor Networks. IEEE Trans. on Mob. Comp. 4, 458-473 (2005)

14. Hou, Y.T., Shi, Y., Sherali, H.D., Midkiff, S.F.: On Energy Provisioning and Relay Node Placement for Wireless Sensor Networks. IEEE Trans. on Wireless Comm. 4, 2579-2590 (2005)

15. Efrat, A., Har-Peled, S., Mitchell, J.S.B.: Approximation Algorithms for Two Optimal Location Problems in Sensor Networks. In: IEEE BROADNETS 2003, Boston (October 2005)

16. Tang, J., Hao, B., Sen, A.: Relay node placement in large scale wireless sensor networks. Comp. Comm. 29(4), 490-501 (2006)

17. Aoun, B., Boutaba, R.: Clustering in WSN with Latency and Energy Consumption Constaints. Journal of Network and Systems Management 14(3), 415-439 (2006)

18. Youssef, W., Younis, M., Akkaya, K.: An Intelligent Safety-Aware Gateway Relocation Scheme for Wireless Sensor Networks. In: IEEE ICC 2006, Istanbul, Turkey (June 2006)

19. Youssef, W., Younis, M.: Intelligent Gateways Placement for Reduced Data Latency in Wireless Sensor Networks. In: IEEE ICC 2007, Glasgow, Scotland (June 2007)

20. Akkaya, K., Younis, M., Youssef, W.: Positioning of Base Stations in Wireless Sensor Networks. IEEE Comm. Mag. 45(4), 96-102 (2007)

21. Fourer, R., Gay, D.M., Kernighan, B.W.: AMPL, A modeling language for mathematical programming (1993)

22. ILOG CPLEX 10.0 user's manual, http://www.ilog.com/products/cplex/ 